\title{
Deformation Pattern and Energy Absorption Characteristics of A Four- Tube Nested System Under Lateral and Oblique Loadings
}

\author{
Bin $X^{a, b}\left(\mathbb{D}\right.$, Cheng WANG ${ }^{b *}$, Steeve Chung Kim Yuen ${ }^{c}$ \\ a Science and Technology on Transient Impact Laboratory, No.208 Research Institute of China Ordnance Industries, Beijing, China. \\ E-mail: xubin12306@163.com \\ b State Key Laboratory of Explosion Science and Technology, Beijing Institute of Technology, Beijing, 100081, China. E-mail: \\ wangcheng@bit.edu.cn \\ c Blast Impact and Survivability Research Unit (BISRU), Department of Mechanical Engineering, University of Cape Town, Cape Town, \\ Republic of South Africa. E-mail: steeve.chungkimyuen@uct.ac.za \\ * Corresponding author
}

https://doi.org/10.1590/1679-78256550

\begin{abstract}
The present research introduces a new configuration of thin-walled nested tube structure as a possible energy absorber subjected to lateral compression. The nested-tube structures were configured in a system comprising of four tubes arranged in different ways. All the nested systems were quasi-statically compressed in the lateral direction on a universal mechanical tester to obtain their energy absorption characteristics. Two modes of deformation, asymmetric mode referred to as "overlap" and symmetric mode referred to as "sideby-side", were identified in the four-tubes nested systems. The specific energy absorbed by the inverted fourtubes systems was larger than that of the three-tubes nested systems by $20 \%$. The experiments were also modelled using ABAQUSv6.13. Good correlations were obtained for the deformation modes and the corresponding lateral crush force-displacement. The validated model was thereafter used in parametric study to investigate the effectiveness of the nested system under two different boundary conditions; rotation of the specimen by different angles and impact at different angles.
\end{abstract}

\section{Keywords}

Nested-tube system; Deformation mode; Energy absorption capacity; oblique load

\section{Graphical abstract}

\section{Crushing Behaviour of a four nested tubes system}

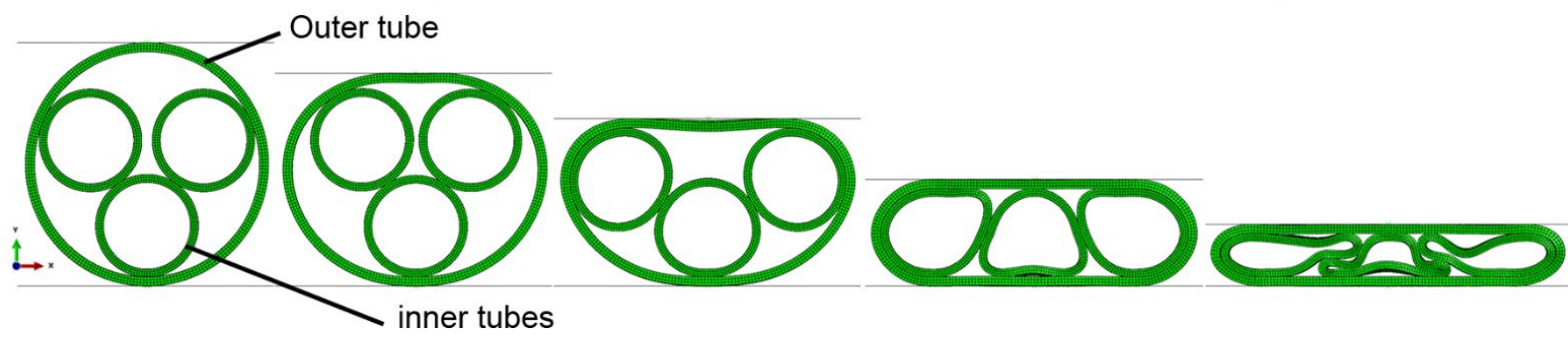




\section{INTRODUCTION}

Thin-walled tubes have been shown to be efficient energy absorbers and are widely used in crashworthiness applications. In the automobile industry, for example, thin-wall tubes are used not only to minimise damage of the vehicle but also to protect the driver and passengers from any injuries [Baroutaji et al., 2017, Lu and Yu, 2003]. Compared with the axially loaded tubes, the thin-walled tubes under lateral crushing load have a smoother force-displacement response due to the distinctive bending failure mode which is better in crashworthiness applications. There have been a number of investigations on the energy absorption capacity and the mechanical response of laterally loaded tubes. Analytical and experimental studies of two-dimensional deformations of rings/short tubes under in-plane loads were reviewed by Lu and $\mathrm{Yu}$ [2003]. The basic energy absorption mechanism of the lateral tubes includes the localised deformation around the plastic hinges. DeRuntz and Hodge [1963] reported on the mechanical behaviours of a thin-walled tube under lateral compression load using a rigid-perfectly plastic material model and a four-hinge deformation mode. Burton and Craig [1963] extended the model developed by DeRuntz and Hodge [1963] by considering a six-hinge deformation mode. However, the force-displacement response of the two theoretical models were similar as a result of analogous force analysis diagram for the undeformed tube. Redwood [1963] updated the model by incorporating material strain hardening effects which were neglected by DeRuntz and Hodge [1963]. However, the theoretical force did not correlate well with the experiments due to inadequate modelling of the stationary plastic hinges for the lateral tubes. Reid and Reddy $[1978,1980]$ later proposed a refined model based on the standard elastic theory replacing the concentrated hinge with an arc for more accurate prediction.

Reid and Reddy [1979] extended their studies to investigate the effect of side constraints so that the diameter of the tube could not increase horizontally. This "closed system" was shown to absorb three times the amount of energy at any given deflection when compared to an open system, tube without any lateral constraints. Xiang et al. [2017] reported on the compression of short tubes between two parallel platens tilted at different angles. The results showed that when the tilt angle increased the value of initial collapse load decreased whilst the overall energy absorption increased. Shim and Stronge [1986] used different packing arrangements to investigate the phenomenon of lateral crushing by successive destabilisation for pattern, modes of tube deformation and force transmitted through the array of tubes. Thin-walled tubes were arranged in layers with tubes of adjacent layers located either in-line for square packed or offset by one radius for hexagonally-packed. The result of the experiment showed that the mean force of the hexagonally-packed was much higher than that of the square-packed.In a different application for blast mitigation, Xia et al. [2016] and Yuen et al. [2017] introduced sandwich cladding panels consisted of core made with multiple thin-walled tubes arranged in different configurations based on the number of tubes and its spacing arrangements. The results showed that these cladding panels can be optimised for blast mitigation.

In addition to the use of multiple thin-walled tubes arranged in arrays, nested tube system is another way to increase the overall energy absorption capacity of tube. Tubes with different diameters and wall thicknesses can be stacked internally to form a nested tubular system [Morris et al., 2007, Olabi et al., 2008a]. The nested tube system could be used in limited crush zone and absorb more energy than the single tube because nested tube systems can have more crushable elements for the same crush zone. More energy could, thus, be absorbed per unit length compared to a single tube system. Morris et al. [2007] proposed a type of nested systems in which three thin-walled tubes with different diameters were assembled to form a nested tube system. The gap between the components of the nested system caused sequential deformations of the tubes in the system which in turn caused a non-monotonic increase in force with the rise of the deformation distance. Olabi et al. [2008b] made some changes to the previous systems by inserting a solid cylinder in each gap between the components of the nested tube system. The use of the solid small bars in the nested systems was able to eliminate the non-monotonic rise in the force-displacement response of the nested system presented by Morris et al. [2007]. As reported by Reddy and Reid [1979], the energy absorption capacity of the single tube can be enhanced significantly by applying the concept of external constrains. This phenomenon was also been demonstrated in the nested system by Morris et al. [2006] who analysed the responses of constrained nested systems. The nested systems had either inclined and vertical constraints. It was found that the nested systems with inclined constraints absorbed more energy than those without external constraints. Other attempts to improve the energy absorption capacity of the nested system was introduced by Baroutaji et al. [2016], Yu et al. [2017], Chen and Xue [2011]. The performance indicators which described the effectiveness of energy absorbing systems were reported by Baroutaji et al. [2016] comparing the various nested systems. Yu et al. [2017] compared the performance of a three-tube system to a double-tube system and a singe-tube system. The results showed that the three-tube system provided the highest energy absorption efficiency and exhibited the most stable deformation mode.

Based on the previous study on nested tube system by Xu et al. [Xu et al., 2019], in the present study, nested tube systems using different internally stacked groups of circular tubes werereanalysed. The nested-tube structures were configured in a systems comprising four-tubes arranged in four different ways. All nested systems were quasi-statically compressed in the lateral direction on a universal mechanical tester. The lateral crush force-lateral displacement responses and energy absorption capabilities of the different nested systems were compared. Tests were also performed on specimens or top plate rotated by 10 degree to simulate loading conditions at different angles. The effect of 
geometrical and loading parameters on the deformation modes and the energy absorption capacity of these nested systems were further examined using a validated finite element model that was developed in Abaqus v6.13.

\section{QUASI-STATIC EXPERIMENTAL STUDIES}

\subsection{Specimen}

The nested tube system, as illustrated and listed in Table. 1, consisted of thin-walled circular 6063-T6 aluminum alloy tubes with different wall thicknesses and diameters. The length of tubes were $20 \mathrm{~mm}$. $D$ was the outside diameter of the tube and $t$ was the wall thickness. In this application, it was assumed that the manufacturing process i.e. extrusion for the aluminium tubes had insignificant effect on energy absorption. Closed-cell aluminium foam with a nominal density of $0.4 \mathrm{~g} / \mathrm{cm} 3$ were cored from a $200 \mathrm{~mm} \times 200 \mathrm{~mm}$ plate to form a cylinder that was inserted in one of the inner tube of the four-tubes system to provide additional stiffness to the inner tube. No adhesion was used to hold the different tubes and aluminum foam in place. In total five different configurations, listed in Table. 1, were tested. The three-tubes nested system was used a baseline for comparison.

Table. 1 Configurations and dimensions of the nested-system tested

\begin{tabular}{|c|c|c|c|c|c|c|}
\hline \multirow{2}{*}{ Identification } & \multirow{2}{*}{ Arrangement } & \multicolumn{2}{|c|}{ Outer Ring } & \multicolumn{2}{|c|}{ Inner Ring } & \multirow{2}{*}{$\begin{array}{c}\text { Nominal Mass } \\
\text { (g) }\end{array}$} \\
\hline & & $D(m m)$ & $t(\mathrm{~mm})$ & $D(m m)$ & $t(\mathrm{~mm})$ & \\
\hline Three tubes nested system(TT) & & 38 & 1.4 & $13.5 / 22$ & $1.3 / 1.6$ & 16.5 \\
\hline Four tubes nested system(FT) & & 38 & 1.4 & 16 & 1.2 & 16.1 \\
\hline Inverted four-tubes nested system (IFT) & & 38 & 1.4 & 16 & 1.2 & 16.1 \\
\hline $\begin{array}{l}\text { Aluminium foam filled four-tubes nested } \\
\text { system(FFT) }\end{array}$ & & 38 & 1.4 & 16 & 1.2 & 18.2 \\
\hline $\begin{array}{l}\text { Inverted aluminium foam filled four-tubes nested } \\
\text { system(IFFT) }\end{array}$ & & 38 & 1.4 & 16 & 1.2 & 18.2 \\
\hline
\end{tabular}

\subsection{Quasi-static Experiments}

All nested systems were quasi-statically compressed in the lateral direction on the universal mechanical tester (model: UTM5504, capacity:50kN). The specimen was carefully placed in the centre of a fixed bottom platen using cello tape. The top platen was set to move downwards at a constant velocity of $5 \mathrm{~mm} / \mathrm{min}$ to laterally crush the specimen. The experimental set-up is shown in Fig. 1. In all cases, the crushing process was stopped at a compressive force of $20 \mathrm{kN}$ when densification of the nested system occurred to avoid possible damage to the apparatus. In most cases, that was equivalent to a lateral crush distance of about $30 \mathrm{~mm}$. Data acquisition software, connected to the load cell and a computer, recorded the forcedisplacement history of the crushing event.The energy absorbed $\left(E_{\mathrm{a}}^{\mathrm{s}}\right)$ and mean crushing force $\left(P_{\mathrm{m}}\right)$ were calculated using Eqs. 1 and 2. The trapezoidal rule (Eq. 1) was applied to integrate the area under the force-displacement graph and represented the energy absorbed during the quasi-static lateral crushing event. pi $(2 \leq i \leq N)$ and $\delta i(2 \leq i \leq N)$ were the sampling points of the force and the displacement respectively. The maximum value of $N$ is 12800 . The mean crushing force $p_{\mathrm{m}}$ was obtained by dividing the energy absorbed with the lateral crushed distance.

$E_{a}^{S}=\sum_{i=2}^{N} 0.5\left(p_{i}+p_{i-1}\right)\left(\delta_{i}-\delta_{i-1}\right)$

$P_{m}=E_{a}^{s} / \delta$

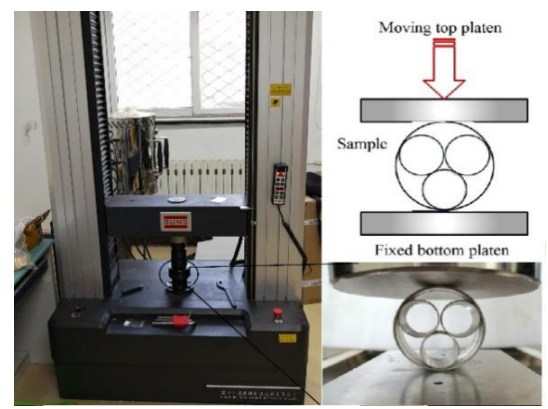

Fig. 1 Experimental arrangement for the quasi-static tests 


\subsection{Results from the quasi-static tests}

A summary of the quasi-static lateral crush tests carried out on the different configurations of the nested tube system is listed in Table 2. A minimum of three tests was performed for each configuration tested. Figs. 2-5 show the typical progressive collapse of different types of nested tube system with its accompanying force-displacement curve. In general, the deformation was characterized by permanent collapse of the tubes with the formation of plastic hinges at different locations along the circumference of both the inner and outer tubes. Various mechanisms of deformation of the tube were observed. SEA is the specific energy absorption which was obtained by dividing the energy absorbed by the mass of the nested tube system, as shown in Eq 3.

Table. 2 Summary of quasi-static test results for different nested tube systems

\begin{tabular}{|c|c|c|c|c|c|c|c|c|c|c|}
\hline \multirow[b]{2}{*}{ Configuration } & \multirow{2}{*}{$\begin{array}{l}\text { Test } \\
\text { No. }\end{array}$} & \multirow{2}{*}{$\begin{array}{l}\text { Weight } \\
\text { (g) }\end{array}$} & \multicolumn{4}{|c|}{ Crush distance: $27 \mathrm{~mm}$} & \multicolumn{4}{|c|}{ Crush distance: $30 \mathrm{~mm}$} \\
\hline & & & $\begin{array}{l}P_{\text {mean }} \\
(\mathrm{kN})\end{array}$ & $\begin{array}{c}E_{\text {absorbed }} \\
(J)\end{array}$ & $\begin{array}{l}\text { SEA } \\
(\mathrm{J} / \mathrm{g})\end{array}$ & $\begin{array}{c}\text { Deviation from } \\
\text { mean }\end{array}$ & $\begin{array}{l}P_{\text {mean }} \\
(\mathrm{kN})\end{array}$ & $\begin{array}{c}\text { Eabsorbed }_{\text {(J) }} \\
\text { (J) }\end{array}$ & $\begin{array}{l}\text { SEA } \\
(\mathrm{J} / \mathrm{g})\end{array}$ & $\begin{array}{c}\text { Deviation from } \\
\text { mean }\end{array}$ \\
\hline & 01 & 16.52 & 2.36 & 63.76 & 3.86 & & 3.07 & 92.0 & 5.57 & \\
\hline & 02 & 16.48 & 2.49 & 67.10 & 4.07 & & 3.19 & 95.3 & 5.78 & \\
\hline & 03 & 16.50 & 2.40 & 64.85 & 3.93 & & 3.17 & 94.2 & 5.71 & \\
\hline 1 & 04 & 16.51 & 2.54 & 68.5 & 4.15 & & 3.34 & 100.2 & 6.07 & \\
\hline & Mean & & 2.45 & 66.03 & 4.00 & $\pm 5 \%$ & 3.19 & 95.43 & 5.78 & $\pm 5 \%$ \\
\hline & 01 & 16.08 & 2.24 & 60.48 & 3.76 & & 2.88 & 86.44 & 5.37 & \\
\hline & 02 & 16.11 & 2.14 & 57.69 & 3.58 & & 2.63 & 78.94 & 4.90 & \\
\hline & 03 & 16.11 & 2.37 & 63.96 & 3.97 & & 3.01 & 90.25 & 5.60 & \\
\hline & 04 & 16.10 & 2.02 & 54.44 & 3.38 & & 2.52 & 75.53 & 4.69 & \\
\hline & 05 & 16.12 & 2.05 & 55.29 & 3.43 & & 2.59 & 77.58 & 4.82 & \\
\hline & Mean & & 2.25 & 60.71 & 3.77 & $\pm 10 \%$ & 2.84 & 85.21 & 5.29 & $\pm 10 \%$ \\
\hline & & & \multicolumn{8}{|c|}{ Side by side } \\
\hline & 01 & 16.10 & 2.99 & 80.67 & 5.01 & & 3.86 & 115.89 & 7.20 & \\
\hline & 02 & 16.08 & 2.90 & 78.33 & 4.87 & & 3.81 & 114.42 & 7.11 & \\
\hline & 03 & 16.11 & 2.97 & 80.21 & 4.98 & & 3.92 & 117.59 & 7.30 & \\
\hline & 04 & 16.11 & 3.37 & 91.03 & 5.65 & & 4.90 & 122.68 & 7.62 & \\
\hline & Mean & & 2.95 & 79.74 & 4.95 & $\pm 5 \%$ & 3.86 & 115.97 & 7.20 & $\pm 5 \%$ \\
\hline & & & \multicolumn{8}{|c|}{ Overlap } \\
\hline IFT & 05 & 16.10 & 2.67 & 72.15 & 4.48 & & 3.20 & 95.96 & 5.96 & \\
\hline & 06 & 16.11 & 2.56 & 69.12 & 4.29 & & 2.87 & 86.08 & 5.34 & \\
\hline & 07 & 16.11 & 2.66 & 71.72 & 4.45 & & 3.60 & 107.88 & 6.69 & \\
\hline & 08 & 16.11 & 2.22 & 59.94 & 3.72 & & 2.48 & 74.48 & 4.62 & \\
\hline & Mean & & 2.63 & 71.00 & 4.41 & $\pm 5 \%$ & 3.22 & 96.64 & 6.00 & $\pm 5 \%$ \\
\hline & 01 & 18.20 & 3.75 & 101.20 & 5.56 & & 4.03 & 121.03 & 6.65 & \\
\hline & 02 & 18.19 & 3.96 & 106.99 & 5.88 & & 4.38 & 131.26 & 7.21 & \\
\hline & 03 & 18.21 & 3.61 & 97.43 & 5.35 & & 4.06 & 121.80 & 6.69 & \\
\hline FFT & 04 & 18.20 & 2.78 & 75.00 & 4.12 & & 3.21 & 96.26 & 5.29 & \\
\hline & Mean & & 3.77 & 101.87 & 5.60 & $\pm 5 \%$ & 4.16 & 124.70 & 6.85 & $\pm 5 \%$ \\
\hline & 01 & 18.21 & 3.35 & 90.50 & 4.97 & & 4.10 & 123.00 & 6.75 & \\
\hline & 02 & 18.20 & 4.00 & 108.12 & 5.94 & & 4.37 & 131.03 & 7.20 & \\
\hline & 03 & 18.19 & 3.58 & 96.61 & 5.31 & & 4.44 & 133.19 & 7.32 & \\
\hline IFFT & 04 & 18.18 & 2.42 & 65.28 & 3.59 & & 3.11 & 93.28 & 5.13 & \\
\hline & Mean & & 3.64 & 98.41 & 5.41 & $\pm 10 \%$ & 4.30 & 129.07 & 7.09 & $\pm 5 \%$ \\
\hline
\end{tabular}

\subsubsection{Results of three-tubes nested system}

The three-tubes nested comprised of two small tubes of different diameters and thicknesses placed inside an outer large tube. Fig. 2 shows the typical deformation process of the three-tubes nested system along with its accompanying lateral crushed force. The response of the three-tubes nested system was typical of what has been previously reported by Baroutaji et al. [2016] and Yu et al. [2017]. As the three-tubes nested system started to collapse, the outer tube deformed simultaneously with the larger inner tube. At this stage, the small inner tube acted as a cylindrical indenter 
compressing the larger inner tube whilst the lateral crushed force plateaued. With increasing lateral crushed distance the larger inner tube cracked at the plastic hinge formed at the top surface with a slight decrease in the lateral crushed force (begin at the crushed distance of $9 \mathrm{~mm}$ ). The inner top tube sunk into the crack surface causing to open. Once the crack in the larger inner tube could not expand anymore (at a crushed distance of $16 \mathrm{~mm}$ ), the small internal tube started to deform causing a gradual increase in the crushed force. From the various stages of the deformation profile of the three-tubes nested system shown in Fig. 2, symmetric collapse was observed. The results of the repeated tests are shown in Fig. 2. No significant experimental variation was observed. All the tests exhibited similar crushed behaviour. As shown in Fig. 2, a significant decrease in lateral crush force for the TT 1 specimen at $25 \mathrm{~mm}$ of lateral displacement could be found, the reason may be that with increasing lateral crushed distance the top inner tube cracked suddenly at one of the the plastic hinge. No obvious decrease of lateral cursh force for other specimens at the same lateral displacement, That's because the deformation of the top inner tube was not exactly symmetrical, then the location of the plastic hinges and their evolution was not exactly the same, that should be point out is the crush force of the specimen 4 also experienced a slight decrease when the lateral displacement was about $25 \mathrm{~mm}$.

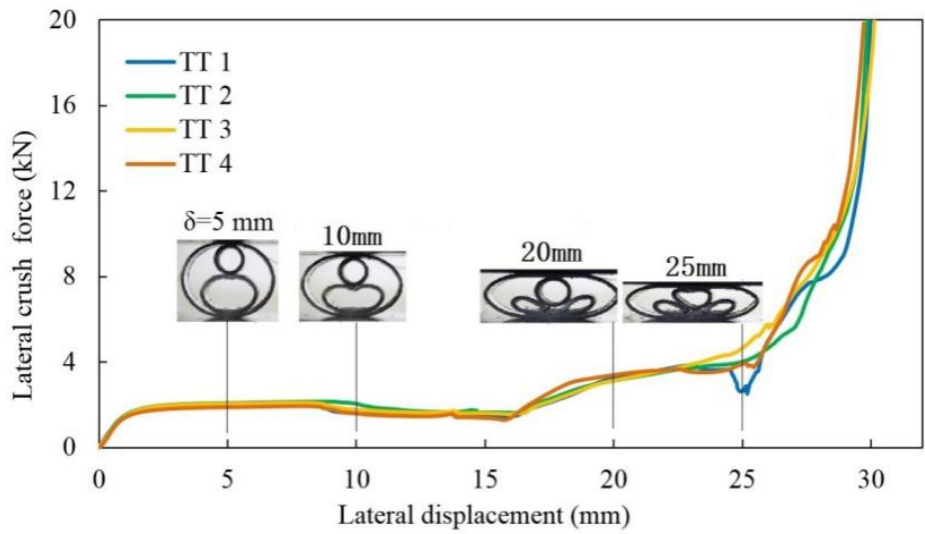

Fig. 2 Typical progression of lateral crush and crushed force vs lateral displacement curves for TT nested systems

\subsubsection{Results of the four-tubes nested system}

The four-tubes nested system is a newly developed system that comprised of three tubes of the same dimensions placed inside a larger outer tube. The three inner tubes were stacked in such a way that their centers formed a triangular shape. In this testing configuration, the top apex of the three inner tubes was located at the compressing end of the fourtubes nested system. Fig. 3 shows the typical deformation process of the nested system and the corresponding lateral crush force with respect to its crushed distance. As the top platen moved downwards the larger outer tube deformed with little deformation observed in the top inner tube. A gentle rise in the lateral crushed force was noted. The two bottom inner tubes were forced sideways inside the outer tube as the compression progressed. The top inner tube was eventually forced down such that all the centers of the tubes were aligned horizontally. Further compression caused all four tubes to deform simultaneously. At this stage symmetry was no longer maintained as the overlapping of inner tubes occurred on either side, observed in repeated tests. This type of asymmetric deformation mode was henceforth referred to as the "overlap" mode. As the structure densified a steeper rise in the lateral crush force was observed. Small experimental variation was observed in the repeated tests, as demonstrated by the lateral crush force-displacement curves shown in Fig. 3.

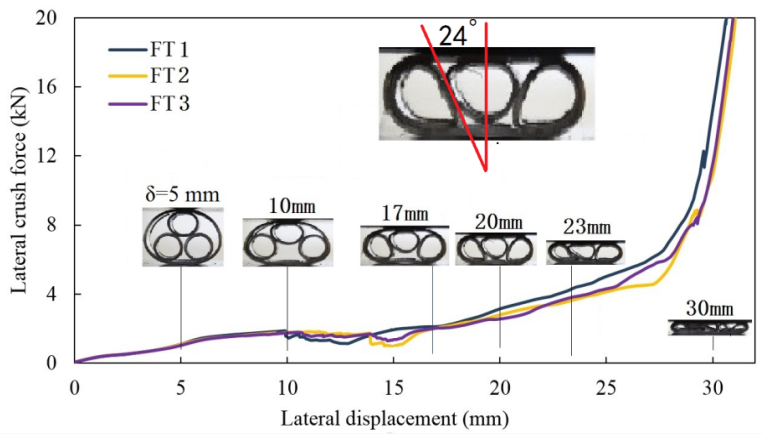

Fig. 3Typical progression of lateral crush and crushed force vs lateral displacement curves for four-tubes nested systems 


\subsubsection{Results of the inverted four-tubes nested systems}

The inverted four-tubes nested system was similar to the four-tubes nested system. However, in this testing configuration, the four-tubes nested system was rotated by 180 degree so that the apex of the three inner tube was now located on bottom of the system. Fig. 4 shows the typical deformation process of the inverted four-tubes nested system along with its accompanying lateral crush force with respect to its lateral displacement. Two different crushing processes were observed in a number of repeated tests. Initially, the lateral crush were similar in all cases. The outer tube started to crush forming an elliptical shape with a fairly gentle increase in lateral crush force. The top two inner tubes then moved sideways in the now available space as the inverted four-tubes nested system was compressed. A small dip in the lateral crushed force was observed (between a crush distances of about $6-7 \mathrm{~mm}$ ) as the top two inner tubes slipped sideways before the lateral crush force was observed to increase steadily. This deformation mode was thus referred to as "side by side". Once the crush distance reached about $15 \mathrm{~mm}$ the lateral crushing proceeded in two possible ways with a steeper increase in lateral crush force.

In one instance, the inverted four-tubes nested systems crushed in a similar way as the non-inverted four-tubes nested system, as expected since the same loading conditions were applied. The only differences were the location of the moving and fixed ends in relation to the inner tubes. The horizontal alignment of the centers of all the tubes was the noted difference in this case. The two top inner tubes did not move down enough to align with the centers. As the compression progressed the bottom inner tube and one of the side inner tube collapsed so that their surfaces overlapped while the lateral crush force plateaued. The nested system crashed in an "overlap" mode eventually and symmetry was no longer conserved.

In the other instance, as the lateral crush progressed the top two inner tubes dropped on either sides of the bottom inner tube aligning the centers of all the tubes horizontally forming a "side-by-side" mode. As the top platen progressed downwards the three inner tubes began to collapse with the outer tube symmetrically with increasing lateral crush force before plateauing. The inner tubes crushed like constrained tubes under lateral compression. The formation of cracks in the middle inner tube caused sudden drop in the lateral crush force. Densification caused sharp increase in the lateral crush force.

Fig. 4 shows the lateral crush force vs displacement curves for all the tests carried out in this configuration. Generally there were little variation in forces up to a displacement of about $17 \mathrm{~mm}$. The noticeable differences were observed in the following stages of the compression as a result of either "overlap" mode of failure or "side-by-side" mode of failure. Higher lateral crush force was observed in the "side-by-side" mode compared to the "overlap" mode.

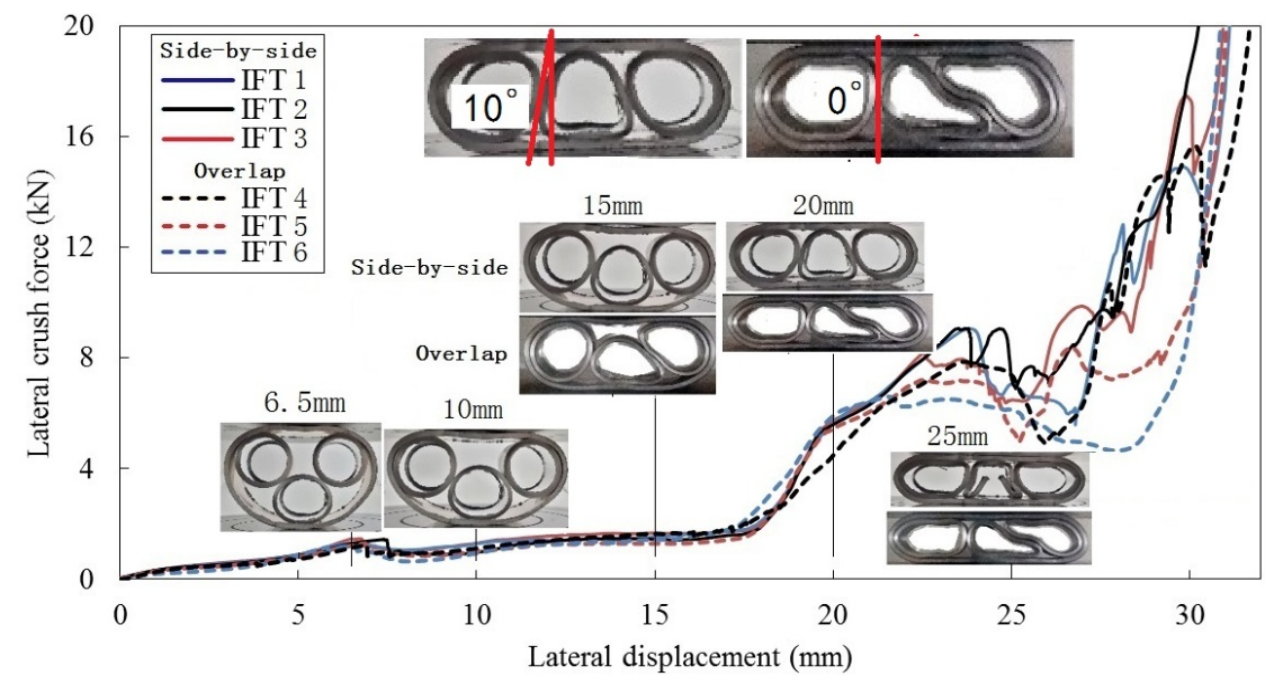

Fig. 4 Typical progression of lateral crush and crushed force vs lateral displacement curves for IFT nested systems

\subsubsection{Results of foam-filled four-tubes nested and inverted foam-filled four-tubes nested systems}

In order to avoid the "overlap" mode of the four-tubes nested system, the middle inner tubes of the system was stiffened by filling it with closed-cell aluminium foam. The choice of the middle inner tube also meant that symmetry was maintained. The weight of the aluminium foam core was nominally $2 \mathrm{~g}$. The foam-filled four-tubes nested system was tested in the two set-ups, as shown in Table. 1. Fig. 5 shows the typical deformation process of the foam-filled four-tubes and inverted foam-filled four-tubes nested systems along with their accompanying force and energy response with 
respect to its crushed distance. Irrespective of how the specimen was located, in both cases, the foam-filled four-tubes and inverted foam-filled four-tubes nested systems behaved in a similar manner, as expected. Both specimens began to collapse symmetrically with the outer larger tube forming an elliptical shape with the top or bottom tube moving in between the other two empty tubes, aligning all the centers of the tubes horizontally, to form a "side-by-side" mode. The lateral crush force gradually increased as compression started. A small dip in the force was observed when the foam filled inner tube slipped sideways of the other inner tubes. When the "side by side" model was formed, failure progressed with all four tubes crushing simultaneously with increasing lateral crush force. No significant experimental variations, as shown in Fig. 5, were observed in both crushing scenarios. In both crushing configurations, similar mean crushed forces (Eq 2, $3.7 \mathrm{kN}$ ) and energy absorbed (100 J) were obtained, the mean crush force and energy absorbed were calculated when the lateral displacement was $27 \mathrm{~mm}$.

In the compression test of the IFT nested system, when the centre of three inner tubes were aligned horizontally, the contact surface of the bottom inner tube and other inner tubes was basically vertical as shown in Fig 4.The deformation of the top inner tube of the FT nested system was much bigger than the bottom inner tube of the IFT nested system before all the centres of inner tubes were aligned horizontally, so the angle of the contact surface was different between the FT nested system and IFT nested system. In the compression test of the FT nested system, when the centres of three inner tubes were aligned horizontally, the contact surface of the top inner tube and other inner tubes was not vertical, or the angle between the contact surface and the compression direction was much bigger than that of the IFT nested system, as shown in Fig 3, that is the main reason why the force-displacement curves of the FT nested system was different to that of the IFT nested system. In the compression test of the IFFT and FFT nested system, no matter what type of nested system was used, both the contact surfaces mentioned before were vertical, or the angle between the contact surface and the compression direction was smaller than that of the FT nested system. In this case, the forcedisplacement curves of two types of foam-filled nested system were similar.

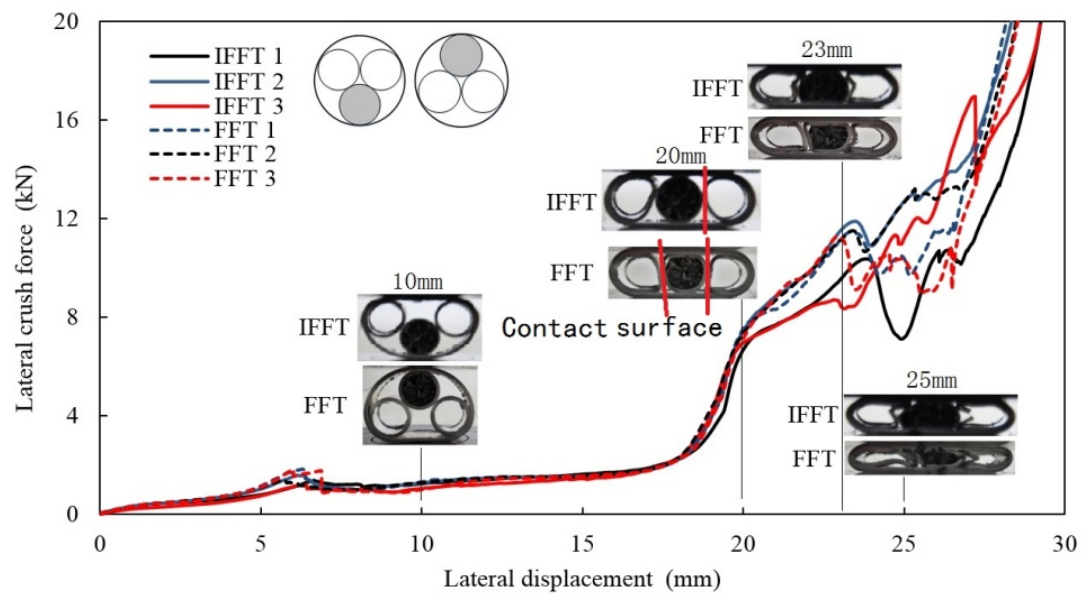

Fig. 5 Typical progression of lateral crush and crushed force vs lateral displacement curves for IFFT and IFT nested systems

2.3.5 Performance of the different nested tube systems

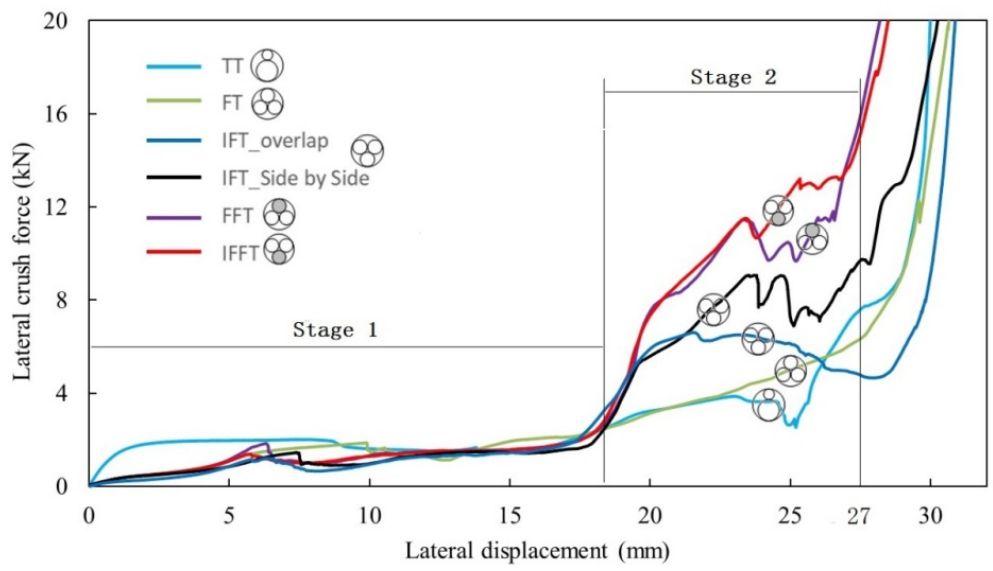

Fig. 6 Graphs comparing the lateral crush force of the different nested tube configurations 
The typical lateral crush forces of the different nested tube configurations are compared in Fig. 6 . In general all the different nested tube systems demonstrated lateral crush force trends that could be divided into two different stages. In the first stage $(0-18 \mathrm{~mm})$, there were generally a gradual increase in the lateral crush force of four tube nested system as it was mostly the outer tube deforming. The three-tubes nested system exhibited higher lateral crush force in this early stage because the larger inner tube was also deforming in this initial stage. In the second stage $(18-27 \mathrm{~mm})$, the four-tube nested systems exhibited higher lateral crush force as the inner tubes had displaced and the compression of four tubes was taking place.

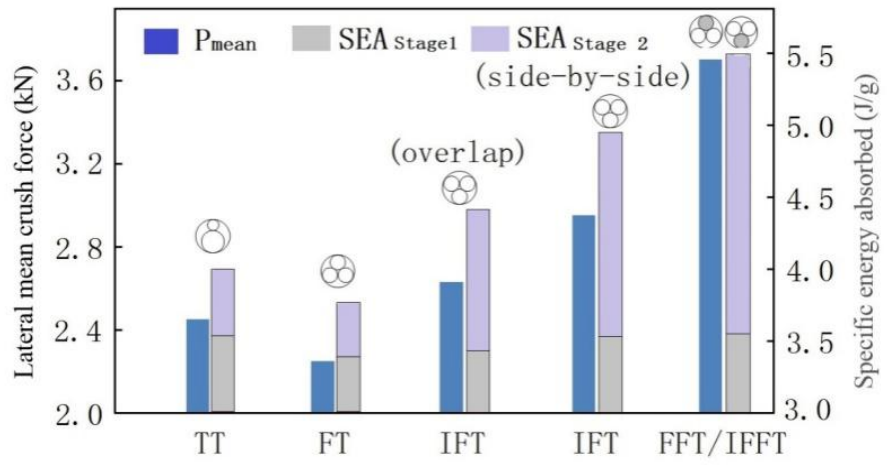

Fig. 7Bar chart comparing the mean lateral crush force of the different nested tube configurations and the mean specific energy absorbed by the different nested tube configuration

Whilst it is important to evaluate the lateral crush force characteristic with lateral displacement, the mean crush force $P_{\mathrm{m}}$ is more valuable in crashworthiness analysis. For a more meaningful comparison, the mean crush force $P_{\mathrm{m}}$ of the different nested systems, listed in Table. 2 and shown graphically in Fig. 7, was compared for the same lateral crush distance of $27 \mathrm{~mm}$. Overall, the four-tubes system exhibited the lowest mean lateral crush force. The mean lateral crushed force of the inverted four-tubes nested systems that collapsed in the side-by-side mode was larger than that of the three-tubes nested systems by $20 \%$. The results showed that the variation in the mean lateral crush force of the inverted four-tubes nested system that collapsed in either the side-by-side or overlap mode was about $\pm 5 \%$. The results from the all the foam filled four-tubes and inverted foam-filled four-tubes nested systems were combined because of their similarities in compression mode. The use of the aluminium foam as a filler material was generally aimed at improving the crashworthiness performance of thin wall tubes system as have been reported in numerous studies on the lateral and axial crushing behaviours and energy absorption capacity of the foam-filled tubes [Shen et al., 2015, Niknejad et al., 2013, Fan et al., 2011, 2013]. However, it must be noted that in this study, the main function of the foam filled tube was to adjust the deformation mode of the four-tubes nested system. It can be observed from the results obtained that the addition of aluminium foam succeeded in consistently providing the side-by-side deformation mode. There was also an increase in the mean lateral crush force by $25 \%$ compare to the inverted four-tubes nested system that collapsed in the "side-by-side" mode.

Specific energy absorption (SEA, Eq.3) was usedto evaluate of the energy absorption ability of all the nested system in a normalised way because of the small differences in the mass of the different nested systems. The energy absorbed by the system $\left(E_{\mathrm{a}}^{\mathrm{S}}\right.$, Eq. 1$)$ was divided by the mass $(m)$ of specimen to obtain the specific energy absorbed. Fig. 7shows the comparison between the SEA for the different nested systems under lateral loading. In the initial compression stage (stage 1, 0-18mm) no noticeable difference was observed for the different four-tubes nested systems. The three-tubes nested system, however, exhibited higher SEA at this stage. In Stage $2(18-27 \mathrm{~mm})$ when the inner tubes of the four-tubes nested systems began to crush, higher SEA was observed for the inverted four-tubes nested systems that collapsed in the "side-by-side" mode. The four-tubes nested system exhibited the lowest SEA. Whilst the inclusion of the aluminium foam in the four-tubes nested system resulted in an increase in mass of about $11 \%$ it also caused an increase SEA by $37 \%$ compare to the three-tubes nested system.

$\mathrm{SEA}=E_{a}^{s} / m$

\subsubsection{Effect of crushing specimens rotated}

So far, the results of the different nested-tubes systems were presented for lateral compression set-up in an ideal symmetric condition at an angle of 0 degree. However, in real-life case scenario impact can occur in any directions. The 
effectiveness at energy absorption of the different nested tubes system was thus tested at the specimens that were rotated at 10 degree. The angle of rotation was determined relative to the vertical line of symmetry. Other angles of rotation were investigated numerically. A summary of the quasi-static lateral crush of the nested systems rotated by $10^{\circ}$ is listed in Table. 3. A minimum of three tests was performed for each configuration tested.The lateral crush force and energy absorbed displacement curves of three types of nested systems are plotted in the graphs shown in Fig. 8. In general, a slight decrease in the lateral crush force and mean lateral crush force was observed with the 10 degree increase in angle of rotation of the specimen. There were no noticeable difference in the magnitude of the lateral crush force of the three-tubes nested system and inverted four-tubes nested system crushed at a rotated angle of 10 degree. Small drop in the lateral crush force was observed for the three-tubes nested nested system as a result of the top inner tube sliding. Step-like increase was observed in the lateral crush force as the inner tubes were crushed independently. The inverted four-tubes nested system crushed with a gradual increase in the lateral crush force. The lateral crush force of the inverted foam-filled four-tubes nested system was similar to the normal compression. Fig. 9 shows the progressive compression of three-tubesnested systems with its mean SEA. As compression was initiated the small inner tube of the three-tubes nested system, shown in Fig. 9, acted as an indenter as in the normal case scenario impinging on the larger inner tube which was compressed in an asymmetric mode. The smaller inner tube eventually slid off the top of the larger inner tube. The inverted four-tubes nested system crushed in the overlap mode, whilst the inverted aluminium foam filled four-tubes nested system crushed in a side-by-side mode, which was similar to the normal compression. The mean SEA of the inverted aluminium foam filled four-tubes nested system reduced by $0.2 \%$ with the 10 degree increase in angle of rotation of the specimen. The mean SEA of the inverted four-tubes nested system reduced by $22 \%$ and that of the three-tubes nested system reduced by $15 \%$.

Table. 3 Summary of quasi-static test results for different nested tube systems ( $\delta=27 \mathrm{~mm}$ )

\begin{tabular}{cccccc}
\hline Configuration & Test NO. & $\mathbf{P}_{\text {mean }}(\mathbf{k N})$ & $\mathrm{E}_{\text {absorbed }}(\mathrm{J})$ & SEA $(\mathrm{J} / \mathrm{g})$ & Deviation from mean \\
\hline 01 & 02 & 2.08 & 56.27 & 3.40 & 3.52 \\
\\
\end{tabular}

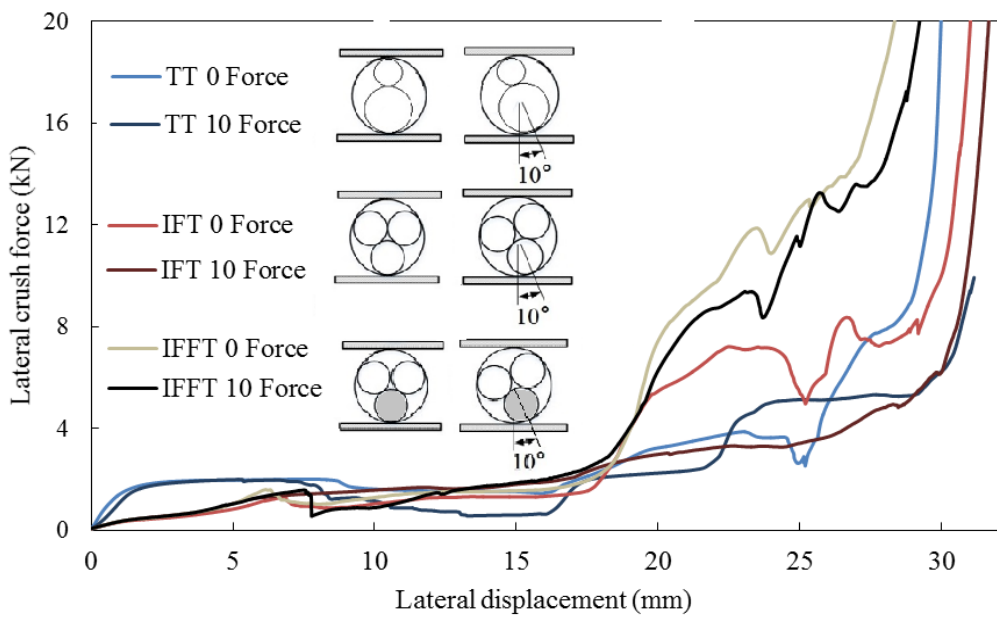

Fig. 8 Typical lateral crushed force vs lateral displacement curve for the nested system rotated by different angles (either 0 or 10 degree) 


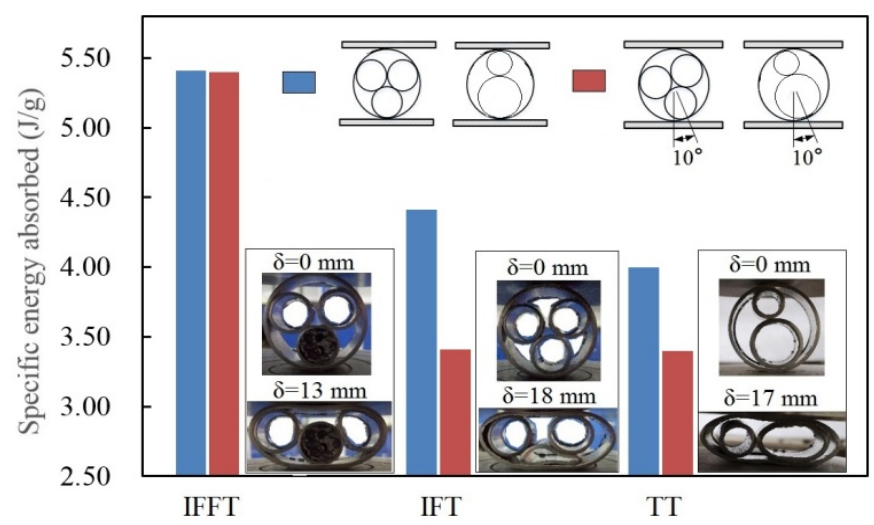

Fig. 9 Graph showing a typical progression of lateral crush and the mean specific energy absorbed $(\delta=27 \mathrm{~mm})$ for the nested systems rotated by 10 degree

\section{DEVELOPMENT OF THE FINITE ELEMENT MODELS}

The explicit non-linear finite element (FE) code ABAQUS v6.13 was used to predict the quasi-static compression of the nested systems. The model consisted principally of the top and bottom platens using two rigid planes and the specimens. The components of the specimens were discretised with full integration explicit solid element consisting of eight nodes having translations, velocities and accelerations in the $x, y$ and $z$ directions at each node. The different tubes were meshed with elements that were, maximum, $0.5 \mathrm{~mm} \times 0.5 \mathrm{~mm} \times 0.5 \mathrm{~mm}$ in size based on the results obtained from previous studies by Olabi et al. [2008b]. The geometric size of the tube used in this research was varied in a big range. In their study, three layers of elements through the wall thickness of any tubes were implemented irrespective of the size of tube.For the different boundary conditions; the top platen was constrained in all rotational and translational degrees of freedom except for translation parallel to the $y$-axis at a constant velocity to simulate the lateral crushing. All translational and rotational degrees of freedom were constrained for the bottom platen.

\subsection{Material Properties for the nested tube system and mesh sensitivity}

The Johnson and Cook material model [Johnson et al., 1985] was used for the nested tube system. The material model describes the flow stress as a function of strain, strain rate, and temperature. The material model also assumes the strength of the material is isotropic and independent of mean stress. The constants $A, B, n, C, m$, and $\dot{\varepsilon}_{0}$ are material dependant parameters and may be determined from an empirical fit of flow stress data. The values of $A, B, C$ and $n$, listed in Table 4, were obtained from Bambach et al. [2010] who used similar Aluminium 6063-T6. Strain rate and temperature effects were neglected because the experiments simulated were quasi-static. Strain rate and temperature effects were considered insignificant.

The model for an empty outer tube of the IFT nested system was used for the mesh sensitivity analysis. The results of a mesh sensitivity analysis, listed in Table 5, were compared for plastic deformation energy. In all cases, the nested system was subjected to a lateral compression of $20 \mathrm{~mm}$. The number of elements through the wall thickness of the tubes were varied between 1, 3 and 5 . The results showed that three layers of elements through the wall thickness culminated to an error of $6 \%$ which was deemed adequate for the numerical model.

Table. 4 Material properties of the tubes and the Johnson-Cook material model parameters [Bambach et al. 2010]

\begin{tabular}{ccccccc}
\hline Young's Modulus (GPa) & $\begin{array}{c}\text { Density } \\
\left(\mathrm{kg} / \mathrm{m}^{3}\right)\end{array}$ & Poisson'sRatio v & $\boldsymbol{A}(\mathrm{MPa})$ & $\boldsymbol{B}(\mathrm{MPa})$ & $\boldsymbol{n}$ & $\boldsymbol{C}$ \\
\hline 82.3 & 2700 & 0.33 & 209 & 353 & 0.055 & 0.0036 \\
\hline
\end{tabular}

Table. 5 Mesh sensitivity analysis of tubes with different size (compression distance is $20 \mathrm{~mm}$ )

\begin{tabular}{lcccc}
\hline & Experimental test & $\begin{array}{c}\text { Numerical simulation One } \\
\text { layer }\end{array}$ & Three layers & Five layers \\
\hline Outer tube $E_{\text {absorbed }}(J)$ & 14.68 & 13.02 (Cumulative error & 13.80 (Cumulative error & 13.97 (Cumulative error \\
& & $12 \%)$ & $6 \%)$ & $5 \%)$ \\
\hline
\end{tabular}




\subsection{Contact properties and Validation of the FE model}

An automatic surface contact algorithm was implemented to simulate the contact between the different components (top platen, nested tube system and bottom platen). Two interaction parameters were defined for the model assembly. The first one assigned a coefficient of friction to the tangential behaviour while the second one assigned the "hard-contact normal" behaviour which allowed separation after contact. There was no significant effect of the coefficient of friction on the predicted results of the three-tubes nested system, shown in Fig. 10(a), as there was little interaction and movement of the tubes. The value of the coefficient of friction was, however, found to affect deformation mode of the four tubes nested tube system and consequently its lateral crush force - displacement curves as a result of the interactions and movements of the inner tubes. Fig. 10(b) shows the effects of the coefficient of friction on the lateral crush force - displacement curve on the initial compression stage (include a lateral crush distance) of the nested tubes system. When the displacement reached about $6 \mathrm{~mm}$, the static friction force between inner tubes reached a local maximum value before a small dip in the lateral crushed force was observed (between the crush distances of about 6-7 mm) as the top two inner tubes began to slip sideways. The peak force during this area was determined by the friction coefficient. The coefficient of friction used in the simulation was obtained by comparing the predicted forcedisplacement with the experiments. A coefficient of friction of 0.12, as shown in Fig. 10(b), was found to be in better agreement with the experiments and thus adopted for all the rest of the simulations.

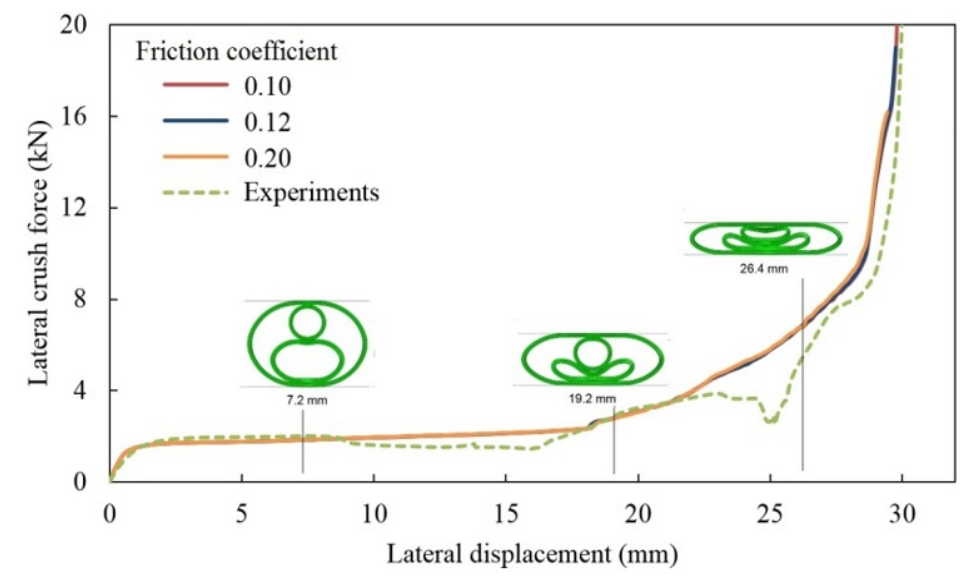

(a) the predicted force-displacement curve of different friction coefficients with experiment for the three-tubes nested system

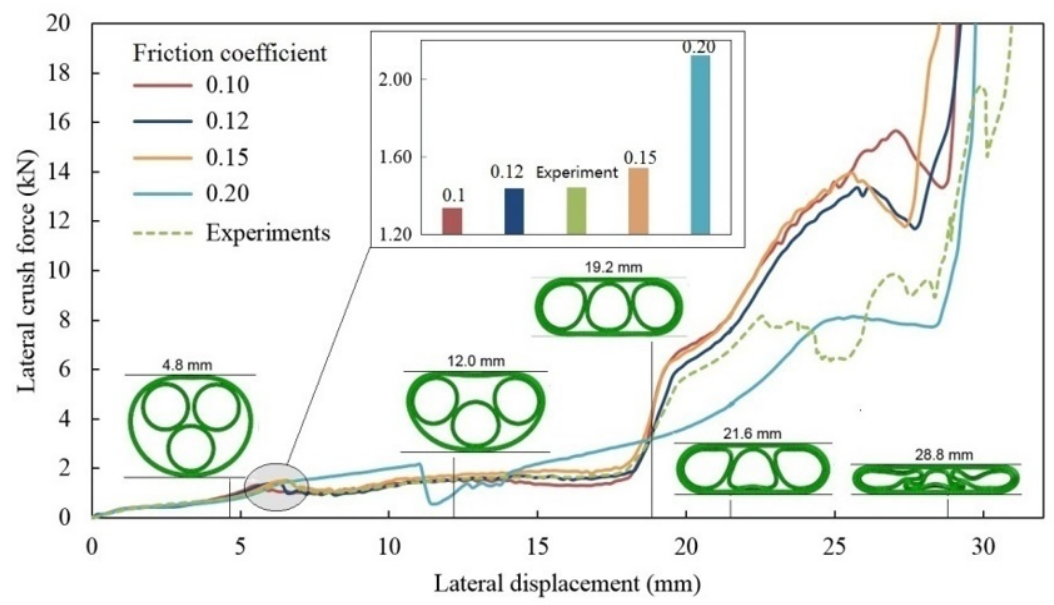

(b) the predicted force-displacement curve of different friction coefficients with experiment for the inverted four-tubes nested system

Fig. 10 Effects of the coefficient of friction on the lateral crush force-lateral displacement curve

The predicted transient compression of the three-tubes nested system is shown in Fig. 10(a). The prediction given by the FE results for the transient deformation compared well with the experiment. The deformation modes predicted by the FE model were compared with those from the experiments for the inverted four-tubes nested system, as displayed in Fig. 10(b). There was very good agreement on the lateral crush force up to a crush distance of about $23 \mathrm{~mm}$ when the top inner tube slid down to form the "side-by-side" mode. Thereafter, the FE model over-predicted the lateral crush 
force because of the omission of a failure model. Cracks were observed in the experiments which caused drop in the lateral crush force. The cumulative error of SEA of simulation compare to experiment in two different displacement is listed in Table. 6.

Table. 6 Cumulative error of SEA of simulation compare to experiment

\begin{tabular}{ccccc}
\hline Configuration & $\begin{array}{c}\text { SEA }(\delta=20 \mathrm{~mm}) \\
\text { simulation/experiment }\end{array}$ & Cumulative error & $\begin{array}{c}\text { SEA }(\delta=27 m m) \\
\text { simulation/experiment }\end{array}$ & $\begin{array}{c}\text { Cumulative } \\
\text { error }\end{array}$ \\
\hline 8$)_{T T}$ & $2.72 / 2.42(\mathrm{~J} / \mathrm{g})$ & $11 \%$ & $4.75 / 4.00(\mathrm{~J} / \mathrm{g})$ & $18 \%$ \\
$88_{\mathrm{IFT}}$ & $1.94 / 2.07(\mathrm{~J} / \mathrm{g})$ & $6 \%$ & $5.97 / 4.95(\mathrm{~J} / \mathrm{g})$ & $21 \%$ \\
\hline
\end{tabular}

\subsection{Parametric Studies}

Table. 7 Summary of predicted results for nested tube systems impacted at different angles

\begin{tabular}{cccc}
\hline & Angle of rotation & \multicolumn{2}{c}{ Crush distance: 28mm } \\
\cline { 3 - 4 } Configuration & $0^{\circ}$ & $\mathbf{P}_{\text {mean }}(\mathbf{k N})$ & 109.2 \\
\hline
\end{tabular}

Overall, it was evident that the numerical results predicted by present finite element model showed good correlation with those from experimental results, shown in Fig. 10b and listed Table. 6 . The omission of a failure criteria to predict tearing and cracks in the specimen was the weakness of the FE model which slightly over predicted the lateral crush force. Nevertheless, satisfactory agreement was met indicating that the present finite element model was adequate and valid and, therefore, could be used for the subsequent analyses.

Two parametric studies, as indicated in Table. 7, were carried out to investigate the effects of impact at different angle of rotation. In one scenario, the nested tube system was rotated by different angles whilst the crush load was still applied in the vertical direction. In the other scenario, the top platen was rotated so that it impacted the specimen at different angles. The angles of rotation of either the specimen or the top platen were set at $10^{\circ}, 20^{\circ}, 30^{\circ}$ and $45^{\circ}$. In both cases, the specimens were fixed in the x-direction at the point of contact between the outer surface of the outer tube and the top surface of the bottom platen to prevent the specimens from going sideways. Both sets of results were compared to the case where the top platen impacted the specimen in a normal direction, refer to as $0^{\circ}$. In these studies, only the inverted four-tubes nested system was investigated. The predicted transient responses for both cases investigated are shown Tables 8 and 9.

\subsubsection{Effect of rotating the specimen}

When the specimen was rotated by $10^{\circ}$, as the lateral crush progressed the top two inner tubes dropped on either sides of the bottom inner tube aligning the centers of all the tubes horizontally forming a "side-by-side" mode. At this stage, symmetrical deformation mode of the bottom inner tube was converted into asymmetrical pattern. As the compression progressed the bottom inner tube and one of the side inner tube collapsed so that their surfaces overlapped. When the specimen was rotated by either $20^{\circ}$ or $30^{\circ}$, the nested system crushed in a similar way. Two inner tubes were crushed on top of each other equivalent to a system in series whilst the third tube slid to the one side and only contributing to energy absorption at a later stage. When the specimen was rotated by $45^{\circ}$, the horizontal alignment of the centers of all the tubes was the noted difference to the specimen was rotated by $10^{\circ}$. The two top inner tubes did not move down enough to align with the centers. As the compression progressed the surfaces of the bottom inner tube and one of the side inner tube overlapped. 
Table. 8 Predicted transient crush response of inverted four-tubes nested system rotated at different angles or crushed at different angles

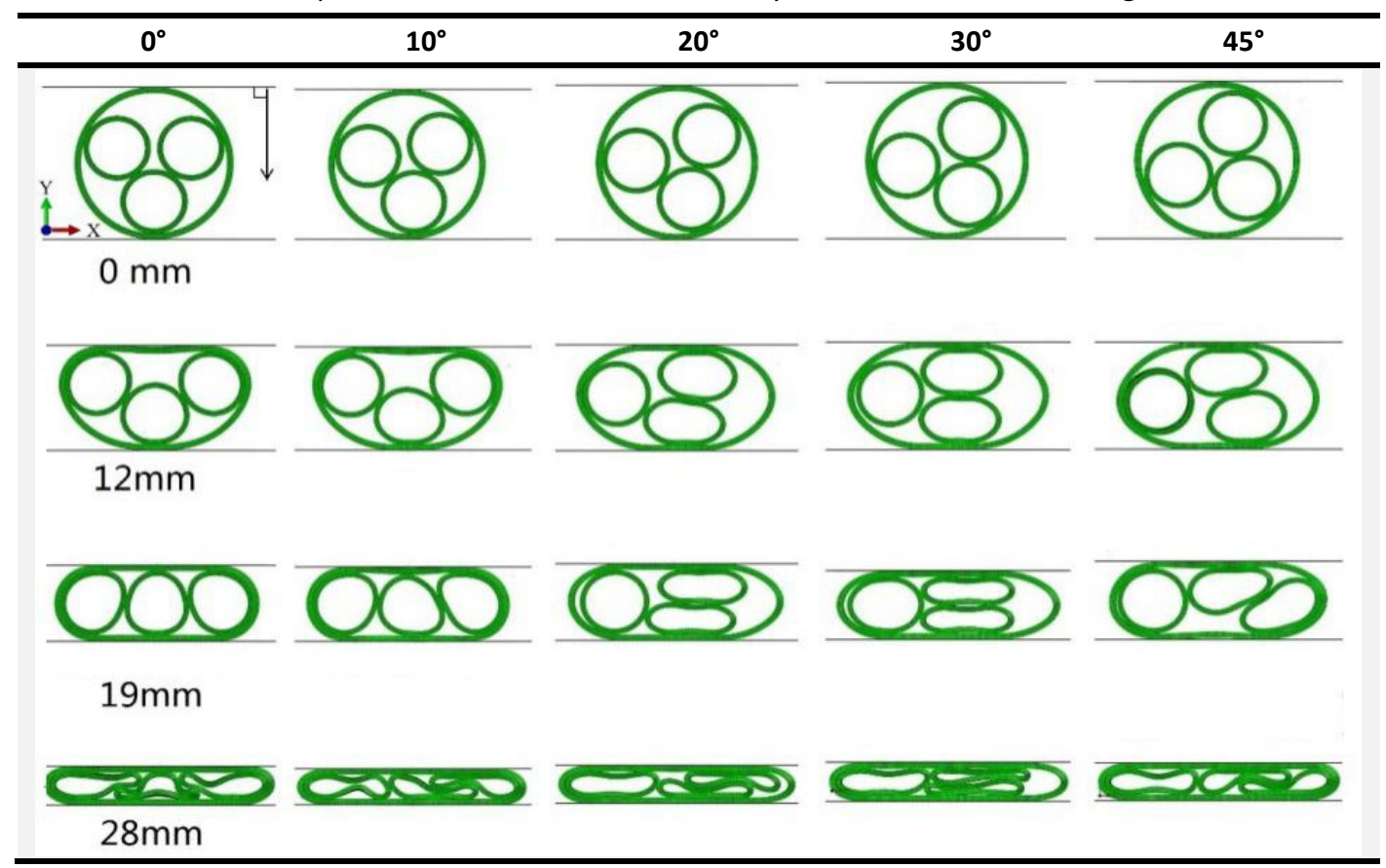

\subsubsection{Effect of rotating the impact direction}

When the top platen was rotated so that it impacted the specimen at different angles. The direction of travel of the top platen was parallel to the normal of the platen surface. The lateral crush force on the normal direction was used to predict the energy absorption of the nested systems. When the platen was rotated by 10, shown in Table.9, "side-by-side" mode of deformation was initiated. Because of the asymmetric deformation, overlap mode of failure subsequently occurred with two inner tubes crushing on top of each other. As the angle of rotation of the top platen was increased, the "side-by-side" mode of deformation was no longer observed as the two inner tubes crushed on top of each other. At an angle of rotation of $30^{\circ}$ the specimen was hardly compressed due to the interaction of two plates. The top platen made contact with the bottom platen limiting the crush distance. The inner tubes crushed in an oval shape with no significant compression.

Table. 9 Predicted transient crush response of inverted four-tubes nested system crushed at different angles

(10)




\subsubsection{Performance of the inverted four-tubes nested systems under oblique load}

The predicted lateral crush force-displacement curves and energy absorbed for the two oblique loading scenarios is shown in Figs. 11 and 12 and summarised in Table 7. For low angles of rotation $\left(10^{\circ}\right.$ and $\left.20^{\circ}\right)$ no significant differences was observed in the lateral crush force and energy absorption when either the specimen or the top platen was rotated. There was, however, significant reduction in the lateral crush force and the subsequent mean lateral crush force and energy absorbed when the specimen or the top platen was rotated compared to compression in the normal direction at $0^{\circ}$. This was due to not only a change in deformation mode but also the least resistant structure for compression. At higher angle of rotation ( $20^{\circ}$ and above) there were no significant variation in the lateral crush force. Consequently, there was no significant changes in the amount of energy absorbed due to the limited displacement for compression.

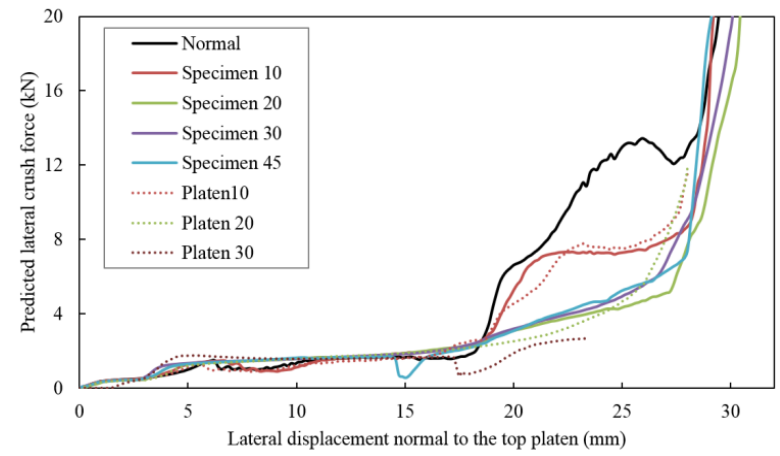

Fig. 11 Predicted lateral crush force for the inverted four-tubes nested system compressed with different boundary conditions

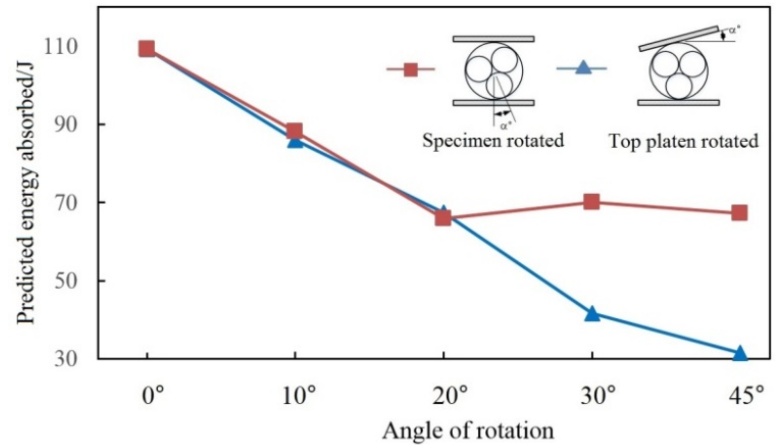

Fig. 12 Predicted energy absorbed for the inverted four-tubes nested system compressed with different boundary conditions

\section{Conclusions}

Experimental investigation into the compression of a novel four-tube nested systems arranged in different ways have been conducted and compared to the three-tube nested system. The lateral crush force-lateral displacement responses and energy absorption capabilities of the different nested systems were compared. Two types of failure modes, "side-by-side" and "overlap" were observed in the deformation of the four-tubes nested system. During the crushing process the inner tubes were compressed with constrained side-by-side conditions. Higher lateral crush force was observed and more energy was absorbed during this stage. The inclusion of the aluminium foam inside one of the inner tubes of the four-tubes nested system consistently provided the side-by-side deformation mode which was the more efficient energy absorber.

The experiments were also modelled using FE method. The simulation results correlated well with the experiments for both crush mode and lateral crush force-displacement characteristics. The validated numerical model was used to conduct two parametric studies to investigate the effects of impact at different angles of rotation. In one scenario, the nested tube system was rotated and in the other scenario, the impact platen was rotated. Although a significant reduction in the energy absorbed when the specimen or the top platen was rotated. In the first scenario, the nested system exhibit excellent energy absorption performance than the second scenario. Such energy absorber could find its application in the engineering fields for enhanced crashworthiness. 


\section{Acknowledgement}

This research is by the Joint Funds of the National Natural Science Foundation of China (Grant No.U20A2071). The opening project number KFJJ17-06M of State Key Laboratory of Explosion Science and Technology, Beijing Institute of Technology.

Author Contributions: B Xu, Conceptualization; Experiments and simulations; review and editing; C Wang, Experiments and simulations; review and editing; Funding acquisition; SCK Yuen: Original draft, review and editing.

Editor: Marcílio Alves

\section{References}

A. Baroutaji, M. Sajjia, A.G. Olabi. [2017]. On the crashworthiness performance of thin-walled energy absorbers: recent advances and future developments. Thin-Walled Structures 118: 137-163.

G. X. Lu, T. X. Yu. [2003]. Energy absorption of structures and materials. Energy Absorption of Structures \& Materials. England.

J. A. DeRuntz, P. G. Hodge. [1963]. Crushing of a tube between rigid plates. Journal of Applied Mechanics 30: 391-395.

R. H. Burton, J. M. Craig. [1963]. An investigation into the energy absorbing properties of metal tubes loaded in the transverse direction. B Sc (England) Report.

R. Redwood. [1963]. Discussion of the paper by JA DeRuntz and PG Hodge. Journal of Applied Mechanics 31: $357-358$.

S. R. Reid, T. Y. Reddy. [1978]. Effect of strain hardening on the lateral compression of tubes between rigid plates. The International Journal of Solids and Structures 16: 545-562.

T. Y. Reddy, S. R. Reid. [1980]. Phenomena associated with the crushing of metal tubes between rigid plates. The International Journal of Solids and Structures 16: 512-524.

T. Y. Reddy, S. R. Reid. [1979]. Lateral compression of tubes and tube-systems with side constraints. International Journal of Mechanical Sciences 21: 187-199.

X. M. Xiang, G. Lu, Z. X. Li, Y. Lv. [2017]. Large deformation of tubes under oblique lateral crushing. International Journal of Impact Engineering 110: 138-148.

V. P. W. Shim, W. J. Stronge. [1986]. Lateral crushing in tightly packed arrays of thin-walled metal tubes. International Journal of Mechanical Sciences 28: 709-728.

Z. C. Xia, X. H. Wang, H. L. Fan, Y. C. Li, F. N. Jin. [2016]. Blast resistance of metallic tube-core sandwich panels. International Journal of Impact Engineering 97: 10-28.

S. Chung kim Yuen, G. Cunliffe, M. C.du. Plessis. [2017]. Blast response of cladding sandwich panels with tubular cores. International Journal of Impact Engineering 110: 266-278.

E. Morris, A. G. Olabi, M. S. J. Hashmi. [2007]. Lateral crushing of circular and non-circular tube systems under quasi-static conditions. Journal of Materials Processing Technology 191: 132-135.

A. G. Olabi, E. Morris, M. S. J. Hashmi, M. D. Gilchrist. [2008a]. A: Optimised design of nested oblong tube energy absorbers under lateral impact loading. International Journal of Impact Engineering 35: 10-26.

A. G. Olabi, E. Morris, M. S. J. Hashmi, M.D. Gilchrist. [2008b]. B: Optimised design of nested circular tube energy absorbers under lateral impact loading. International Journal of Mechanical Sciences 50: 104-116.

E. Morris, A. G. Olabi, M. S. J. Hashmi. [2006]. Analysis of nested tube type energy absorbers with different indenters and exterior constraints. Thin-Walled Structures 44: 872-885.

A. Baroutaji, M. D. Gilchrist, A. G. Olabi. [2016]. Quasi-static, impact and energy absorption of internally nested tubes subjected to lateral loading. Thin-Walled Structures. 98: 337-350. 
Z. L. Yu, P. Xue, Z. Chen. [2017]. Nested tube system applicable to protective structures against blast shock. International Journal of Impact Engineering102: 129-139.

Z. Chen, P. Xue. [2011]. Energy-absorbing performance of nested tube systems under compression load, engineering plasticity and its applications. Proceeding of the 10th ASIA-PACIFIC Conference. WuHan China: World Scientific, 13715-13717.

Bin Xu, Cheng Wang, Wenlong Xu. [2019]. An efficient energy absorber based on fourfold-tube nested circular tube system. Thin-Walled Structures 137: 143-150.

J. Shen, G. Lu, D. Ruan, C. C. Seah. [2015]. Lateral plastic collapse of sandwich tubes with metal foam core. International Journal of Mechanical Sciences 91: 99-109.

A. Niknejad, H. Assaee, S. A. Elahi, A. Golriz. [2013]. Flattening process of empty and polyurethane foam-filled Eglass/vinylester composite tubes- an experimental study. Composite Structures 100: 479-492.

Z. Fan, J. Shen, G. Lu. [2011]. Investigation of lateral crushing of sandwich tubes. Procedia Engineering 14: 442-449.

Z. Fan, J. Shen, G. Lu, D. Ruan. [2013]. Dynamic lateral crushing of empty and sandwich tubes. International Journal of Impact Engineering 53: 3-16.

G. R. Johnson, W. H. Cook. [1985]. Fracture characteristics of three metals subjected to various strains, strain rates, temperatures and pressures. Engineering Fracture Mechanics 21: 31-48.

M. R. Bambach, X. L. Zhao, H. Jama, [2010]. Energy absorbingcharacteristics of aluminium beams strengthened with CFRPsubjected to transverse blast load. International Journal of Impact Engineering 53: 37-49. 